\title{
Lysosome Associated Protein Transmembrane 4B-24 Is the Predominant Protein Isoform in Human Tissues and Undergoes Rapid, Nutrient-Regulated Turnover
}

\author{
Kecheng Zhou, ${ }^{* \dagger}$ Andrea Dichlberger, ${ }^{* \dagger}$ Elina Ikonen, ${ }^{* \dagger}$ and Tomas Blom ${ }^{* \dagger}$
}

From the Department of Anatomy, ${ }^{*}$ Faculty of Medicine, University of Helsinki, Helsinki; and the Minerva Foundation Institute for Medical Research, ${ }^{\dagger}$ Helsinki, Finland

Accepted for publication

July 2, 2020.

Address correspondence to Tomas Blom, Ph.D., Faculty of Medicine, University of Helsinki, Haartmaninkatu 8, Helsinki, 00140 Finland. E-mail: tomas.blom@helsinki.fi.

\begin{abstract}
Studies of lysosome associated protein transmembrane 4B (LAPTM4B) have mainly focused on the $35-\mathrm{kDa}$ isoform and its association with poor prognosis in cancers. Here, by employing a novel monoclonal antibody, the authors found that the 24-kDa LAPTM4B isoform predominated in most, both healthy and malignant, human cells and tissues studied. LAPTM4B-24 lacks the extreme N-terminus and, contrary to LAPTM4B-35, failed to promote cell migration. The endogenous LAPTM4B-24 protein was subject to rapid turnover with a $t_{1 / 2}$ of approximately 1 hour. The protein was degraded by both lysosomal and proteasomal pathways, and its levels were increased by the availability of nutrients and lysosomal ceramide. These findings underscore the pathophysiological relevance of the LAPTM4B-24 isoform and identify it as a dynamically regulated effector in lysosomal nutrient signaling. (Am J Pathol 2020, 190: 2018-2028; https://doi.org/10.1016/j.ajpath.2020.07.003)
\end{abstract}

The late endosomal/lysosomal protein LAPTM4B was originally identified from a transcript up-regulated in hepatocellular carcinoma, ${ }^{1}$ and its expression has been correlated with poor prognosis in several cancers. ${ }^{2,3}$ The LAPTM4B mRNA contains alternative start codons and may be translated into either a $35-\mathrm{kDa}$ protein (LAPTM4B-35) or a 24-kDa isoform (LAPTM4B-24) lacking amino acids 1 to 91. The LAPTM4B-35-specific N-terminus has been reported to contain two functional amino acid motifs, an SH3-domain binding motif (amino acids 12 to 15) interacting with the $\mathrm{p} 85 \alpha$ subunit of phosphatidylinositol 3-kinase (PI3K) to promote efflux of chemotherapeutic drugs, ${ }^{4}$ and an arginine-rich polybasic stretch (amino acids 52 to 67) interacting with phosphoinositides to regulate autophagy. ${ }^{5}$ Both LAPTM4B isoforms have in common the following motifs: a conserved sphingolipid interaction motif in the third transmembrane span (amino acids 208 to 216) important for mammalian target of rapamycin complex 1 (mTORC1) signaling, ${ }^{6}$ C-terminal lysosomal targeting signals, ${ }^{1,7}$ and PY motifs (amino acids 295 to 298, 311 to 314) required for binding the ubiquitin ligase Nedd4.
LAPTM4B-35 represents the longest open reading frame (ORF) of the LAPTM4B transcript, and is commonly considered to be the major isoform. ${ }^{8}$ Consequently, most investigations have focused on LAPTM4B-35, whereas less is known about LAPTM4B-24. Studies of LAPTM4B have been hampered by a lack of tools to reliably detect the endogenous protein, and mechanistic studies have therefore mainly been conducted in overexpression systems. In cases where commercially available antibodies have been used to detect LAPTM4B, proper validation of the detected isoforms has been lacking. Here, the authors validated a monoclonal LAPTM4B antibody and investigated the expression of LAPTM4B isoforms in a range of cell lines, and in healthy and diseased human tissues. The antibody also enabled the authors to study, for the first time, how the endogenous

Supported by Academy of Finland grants 303771 (T.B.), 266092 (T.B.), 282192 (E.I.), 307415 (E.I.), and 312491 (E.I.); the Sigrid Juselius Foundation (T.B., E.I.); The Liv och Hälsa Foundation (T.B.); and the Integrative Life Science doctoral program of the University of Helsinki (K.Z.).

Disclosures: None declared. 
LAPTM4B protein levels are regulated. Further studies were performed comparing the functional differences between the two protein isoforms. It was found that LAPTM4B-24 and -35 expression induce different cellular phenotypes, and that LAPTM4B-24 is the dominant isoform in both healthy and diseased tissues and cells.

\section{Materials and Methods}

\section{Cell Culture}

The cancer cell lines were from ATCC (Manassas, VA), and healthy fibroblasts were from Coriell Institute (Camden, $\mathrm{NJ}$ ). All cells were grown at $+37^{\circ} \mathrm{C}$ and with $5 \% \mathrm{CO}_{2}$; the full culture conditions are listed in Supplemental Table S1. For mTORC1 activity experiments, cells were starved for 1 hour with Earle's Balanced Salt Solution (EBSS) and subsequently refed with stimulation medium (EBSS/complete medium; 1:1) for the indicated time points. To assess the effect of glucose on LAPTM4B expression, cells were starved for 2 hours or 6 hours in Dulbecco's Modified Eagle Medium containing no glucose (Gibco, Waltham, MA; catalog number 11966025).

\section{Reagents, Antibodies, siRNAs, and Quantitative PCR}

C6-ceramide (Enzo Clinical Labs, Farmingdale, NY; catalog number BML-SL110-0005); C6-sphingomyelin (Larodan, Solna, Sweden; catalog number 56-1083-4); oleic acid (SigmaAldrich, St. Louis, MO; catalog number O-1383); fatty acidfree bovine serum albumin (Sigma-Aldrich; catalog number A3803); oleic acid in complex with fatty acid-free bovine serum albumin (BSA) in 8:1 molar ratio prepared in serum-free Dulbecco's Modified Eagle Medium for oleic acid loading cells. Cholesterol (Sigma-Aldrich; catalog number C8669); methyl beta cyclodextrin (Sigma-Aldrich; catalog number C4555); cholesterol in complex with methyl beta cyclodextrin was prepared in $\mathrm{ddH}_{2} \mathrm{O}$ and subjected to sonication, and then used for cholesterol loading cells. The following reagents were also used: cycloheximide (Sigma-Aldrich; catalog number C-7698); bafilomycin-A1 (AppliChem, Darmstadt, Germany; catalog number 88899-55-2); MG-132 (Calbiochem, San Diego, CA; catalog number 474790); dextran TMR (tetramethylrhodamine) (Thermo Fisher Scientific, Waltham, MA; catalog number D1818). The following antibodies were used: mouse monoclonal anti-LAPTM4B antibody (Atlas Antibodies, Bromma, Sweden; catalog number AMAb91356); mouse monoclonal anti-Flag (M2) antibody (Sigma-Aldrich; catalog number F1804); phospho-p70 S6 Kinase (Thr389) antibody (Cell Signaling Technology, Danvers, MA; catalog number 9205); S6 kinase antibody (Santa Cruz Biotechnology, Dallas, TX; catalog number sc-8418); phospho-4E-BP1 (Thr37/46) antibody (Cell Signaling Technology; catalog number 2855); 4E-BP1 antibody (Cell Signaling Technology; catalog number 9452); phospho-ULK1 (Ser 757) antibody (Cell Signaling Technology; catalog number 6888); ULK1 antibody (Cell Signaling Technology; catalog number 8054);
phospho-AKT (Ser 473) antibody (Cell Signaling Technology; catalog number 4060); pan-AKT (C67E7) antibody (Cell Signaling Technology; catalog number 4691); goat anti-mouse $\operatorname{IgG}(\mathrm{H}+\mathrm{L})$-HRP conjugate (Bio-Rad Laboratories, Hercules, CA; catalog number 1706516); goat anti-mouse $\operatorname{IgG}(\mathrm{H}+\mathrm{L})$ Cross-Adsorbed Secondary Antibody, Alexa Fluor 488 (Thermo Fisher Scientific; catalog number A-11001). The predesigned Silencer Select LAPTM4B siRNA (5'-GGAUCAGUAUAACUUUUCATT- $3^{\prime}$ ), SMPD1 siRNA (5'-UCACAG CACUUGUGAGGATT-3'), GBA siRNA (5'-GGAAUCGGAUAUAACAUCATT- $3^{\prime}$ ), and Ctrl siRNA were from Ambion (Waltham, MA). All siRNAs were used at a final concentration of $10 \mathrm{nmol} / \mathrm{L}$, and cells were transfected 2 to 3 days by HiPerFect transfection reagent (Qiagen, Hilden, Germany; catalog number 301705).

Quantitative PCR and primers for detecting LAPTM4B and GAPDH were reported previously. ${ }^{9}$

\section{Generation of Knockout Cells and Stable Cell Lines}

LAPTM4B knockout (KO) A431 and ASAH1 KO A431 were generated by CRISPR/Cas9-mediated genome engineering as described previously. ${ }^{6,9}$ Briefly, coding sequences in exon 3 of LAPTM4B (Gene ID: 55,353) and exon 1 of ASAHI (Gene ID: 427) were selected and analyzed for the design of Cas9 nickase targets (CRISPR, http://crispr.mit.edu, last accessed June 12, 2015). sgRNA (single guide RNA) guides were synthesized and subcloned into the sgRNA expression vector using BbsI sites. Cells were cotransfected with Cas9 nickase and sgRNA expressing plasmids using Lipofectamine LTX with PLUS reagent (Invitrogen, Carlsbad, CA; catalog number 15338100). The cells were under puromycin $(1 \mu \mathrm{g} / \mathrm{mL})$ selection for 48 hours post-transfection, and cultured without puromycin for another 4 days. Subsequently, single clones were isolated and verified by Sanger sequencing and Western blotting. For generating cell lines with missense mutations at LAPTM4B-35 and LAPTM4B24 start codon, CRISPR/Cas9 and homology-directed repair methods were used as described previously. ${ }^{10}$ For generating stable LAPTM4B isoform-expressing cell lines, cells were transfected with pEFIRES-p containing LAPTM4B35-3xFlag, LAPTM4B-24-3xFlag, using Lipofectamine LTX with PLUS reagent. Cells were grown in culture medium containing $1 \mu \mathrm{g} / \mathrm{mL}$ puromycin until a resistant cell pool was formed. mCherry-tagged LAPTM4B-35 was transfected in LAPTM4B-24-3xFlag stable cells to generate the double-tagged stable cell line.

\section{Western Blotting}

Cells were washed with phosphate-buffered saline (PBS), scraped in NP-40 lysis buffer (1\% NP-40, $50 \mathrm{mmol} / \mathrm{L}$ HEPES, $250 \mathrm{mmol} / \mathrm{L} \mathrm{NaCl}, 5 \mathrm{mmol} / \mathrm{L}$ EDTA, proteinase inhibitor cocktail, $\mathrm{pH}$ 7.4), incubated on ice for 10 minutes, and cleared by centrifugation at $12,000 \times g$ for 10 minutes at $4^{\circ} \mathrm{C}$. For 
detecting protein phosphorylation, cells were washed with PBS, scraped in SDS boiling buffer (2.5\% SDS, $250 \mathrm{mmol} / \mathrm{L}$ Tris/ $\mathrm{HCl} \mathrm{pH} 6.8$, including $50 \mathrm{mmol} / \mathrm{L} \mathrm{NaF}, 10 \mathrm{mmol} / \mathrm{L}$ b-glycerophosphate, $0.5 \mathrm{mmol} / \mathrm{L}$ DTT, $0.5 \mathrm{mmol} / \mathrm{L} \mathrm{PMSF}$ ), and lysates were boiled at $98^{\circ} \mathrm{C}$ for 10 minutes, and cleared by centrifugation at $12,000 \times g$ for 10 minutes at $4^{\circ} \mathrm{C}$. Equal amounts of proteins were resolved on 12\% Mini-Protean TGX Stain-Free gels (Bio-Rad Laboratories; catalog number 161-0185) and transferred onto LF-PVDF (Bio-Rad Laboratories; catalog number 170-4274). Membranes were blocked with $5 \%$ milk or $3 \%$ BSA in TBS containing $0.1 \%$ Tween-20 for 1 hour at room temperature and subsequently probed with primary antibodies (anti-LAPTM4B, 1:1000; anti-Flag M2, 1:1000 to 1:2000; anti-phospho-p70 S6K, 1:1000 to 1:2000; anti-S6K, 1:1000; anti-phospho-4EBP, 1:1000 to 1:2000; anti-4EBP, 1:1000; anti-phospho-ULK1, 1:1000; antiULK1, 1:1000; anti-phospho-AKT, 1:1000; anti-AKT, $1: 1000)$ at $4^{\circ} \mathrm{C}$ overnight. After washing with TBS containing $0.1 \%$ Tween-20, membranes were incubated with secondary antibodies for 45 minutes at room temperature. Membranes were washed, incubated with ECL Clarity (BioRad Laboratories; catalog number 170-5060), and imaged with a ChemiDoc MP Imaging System (Bio-Rad Laboratories; catalog number 17001402). Band intensities were analyzed using Image Lab software version 6.0 (Bio-Rad Laboratories), and were normalized to total protein content quantified with Stain-Free technology (Bio-Rad Laboratories). The total protein in each lane served as loading control.

\section{Surface Biotinylation}

The cell surface biotinylation assay was modified from a previously published protocol. ${ }^{11}$ Briefly, cells on $60-\mathrm{mm}$ plates were rinsed twice with ice-cold PBS containing $0.02 \mathrm{mmol} / \mathrm{L} \mathrm{CaCl}_{2}$ and $0.15 \mathrm{mmol} / \mathrm{L} \mathrm{MgCl}_{2}$. Cell surface proteins were biotinylated for 20 minutes with $0.5 \mathrm{mg} / \mathrm{mL}$ biotin (Sulfo-NHS-LC-Biotin; Thermo Fisher Scientific; catalog number 21335) diluted in PBS. Unbound biotin was quenched with PBS containing $0.1 \mathrm{~mol} / \mathrm{L}$ glycine and $0.3 \%$ BSA. The cells were washed with PBS and suspended in $500 \mu \mathrm{L}$ of lysis buffer $(0.2 \%$ SDS, $2 \%$ NP-40 in PBS, supplemented with protease inhibitor cocktail; SigmaAldrich; catalog number P8340). After a 10-minute incubation at $4^{\circ} \mathrm{C}$, lysates were collected and cleared by centrifugation, and an aliquot $(30 \mu \mathrm{L})$ was taken for immunoblotting with the anti-Flag antibody to analyze protein amount before pulldown. Biotinylated proteins from the rest of the lysate were captured with $20 \mu \mathrm{L}$ of streptavidin agarose beads (Sigma-Aldrich; catalog number S1638) on a rotating mixer $(20 \mathrm{rpm})$ overnight at $4^{\circ} \mathrm{C}$. Postpulldown supernatant was collected to assess the amount of non-plasma membrane-associated protein. The beads were washed, and biotinylated proteins were eluted according to the manufacturer's instructions; the proteins were analyzed by Western blotting as described above.
Immunofluorescence Staining and Confocal Microscopy

Immunofluorescence staining was performed as described previously. ${ }^{6}$ Briefly, cells were fixed in $4 \%$ paraformaldehyde in PBS for 20 minutes at room temperature and then quenched with $50 \mathrm{mmol} / \mathrm{L} \mathrm{NH}_{4} \mathrm{Cl}$ for 10 minutes. Cells were washed with PBS and permeabilized with $0.1 \%$ Triton X-100 in PBS for 10 minutes, blocked in 10\% FBS in PBS for 30 minutes, and then incubated with anti-Flag M2 (Sigma-Aldrich; catalog number F1804, 1:400) or anti-LAPTM4B (Atlas Antibodies; catalog number AMAb91356, 1:200) for 45 minutes at $37^{\circ} \mathrm{C}$. Cells were washed in PBS and incubated with secondary antibodies for 45 minutes at $37^{\circ} \mathrm{C}$. Coverslips were washed in PBS, rinsed in $\mathrm{MQ}-\mathrm{H}_{2} \mathrm{O}$ (Merck Millipore, Burlington, MA), and mounted on microscope slides using Mowiol/DABCO (Calbiochem, San Diego, CA; catalog number 475904/ Sigma-Aldrich; catalog number D-2522). For visualizing endosomes, cells was treated with $50 \mu \mathrm{g} / \mathrm{mL}$ dextran TMR (Thermo Fisher Scientific; catalog number D1818) for 2 hours. Images were captured with a Leica TCS SP8 X confocal microscope (Leica Microsystems, Wetzlar, Germany) and analyzed using ImageJ software version $1.50 \mathrm{~b}$ (NIH, Bethesda, MD; http://imagej.nih.gov/ij).

\section{Cell Migration Assay}

Cell migration was measured by using xCELLigene. ${ }^{12}$ Briefly, the CIM-Plate 16 (ACEA, San Diego, CA; catalog number 5665817001) was equilibrated at $37^{\circ} \mathrm{C}$ for 1 hour with serumfree medium in the top chamber and complete medium with $10 \%$ FBS in the lower chamber, after which the plate background was measured. A total of $6 \times 10^{4}$ cells were seeded in the top chamber of each well, and the CIM plate was left at room temperature for 30 minutes, allowing the cells to settle. The plate was transferred to $37^{\circ} \mathrm{C} / 5 \% \mathrm{CO}_{2}$ incubator, and measurements were acquired with an interval of 15 minutes for 27 hours.

\section{Human Tissue Materials}

Human breast cancer and the adjacent non-tumor tissues were from REPROCELL (Beltsville, MD). All breast cancer tissue donors are TNM stage III breast cancer patients. Samples were lysed using a Bullet Blender 24 Gold (Next Advance, Troy, NY; catalog number BB24-AU), according to the cancer tissue homogenization protocol. Briefly, the tissue samples were manually cut into pieces. The samples were washed three times with $1 / 2$ tube volume of PBS to remove surface contaminants. The samples were then placed in tubes with homogenization beads (3.2-mm stainless steel beads; Next Advance; Cat \#SSB32; and 3.5-mm stainless steel UFO beads; Next Advance; catalog number SSUFO35), add radioimmunoprecipitation assay lysis buffer $(25 \mathrm{mmol} / \mathrm{L}$ TRIS/ $\mathrm{HCl}, 150 \mathrm{mmol} / \mathrm{L} \mathrm{NaCl}, 1 \% \mathrm{NP}-40,0.1 \% \mathrm{SDS}, 0.5 \%$ $\mathrm{NaDoc}, 1 \mathrm{mmol} / \mathrm{L}$ EDTA, adjust $\mathrm{pH}$ to 7.6 ) with protease 
A

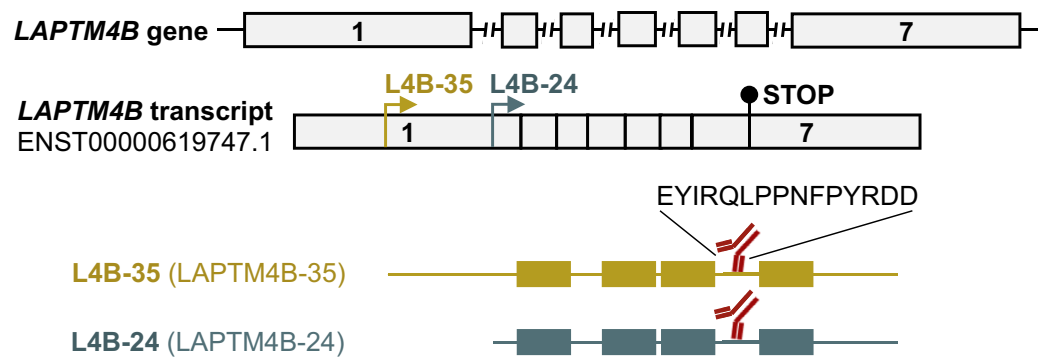

B

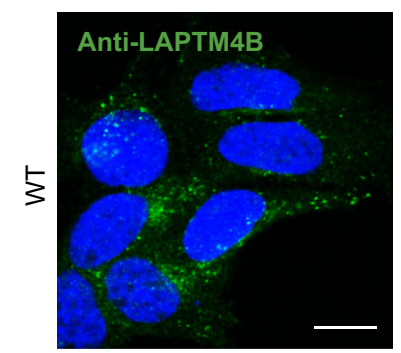

C
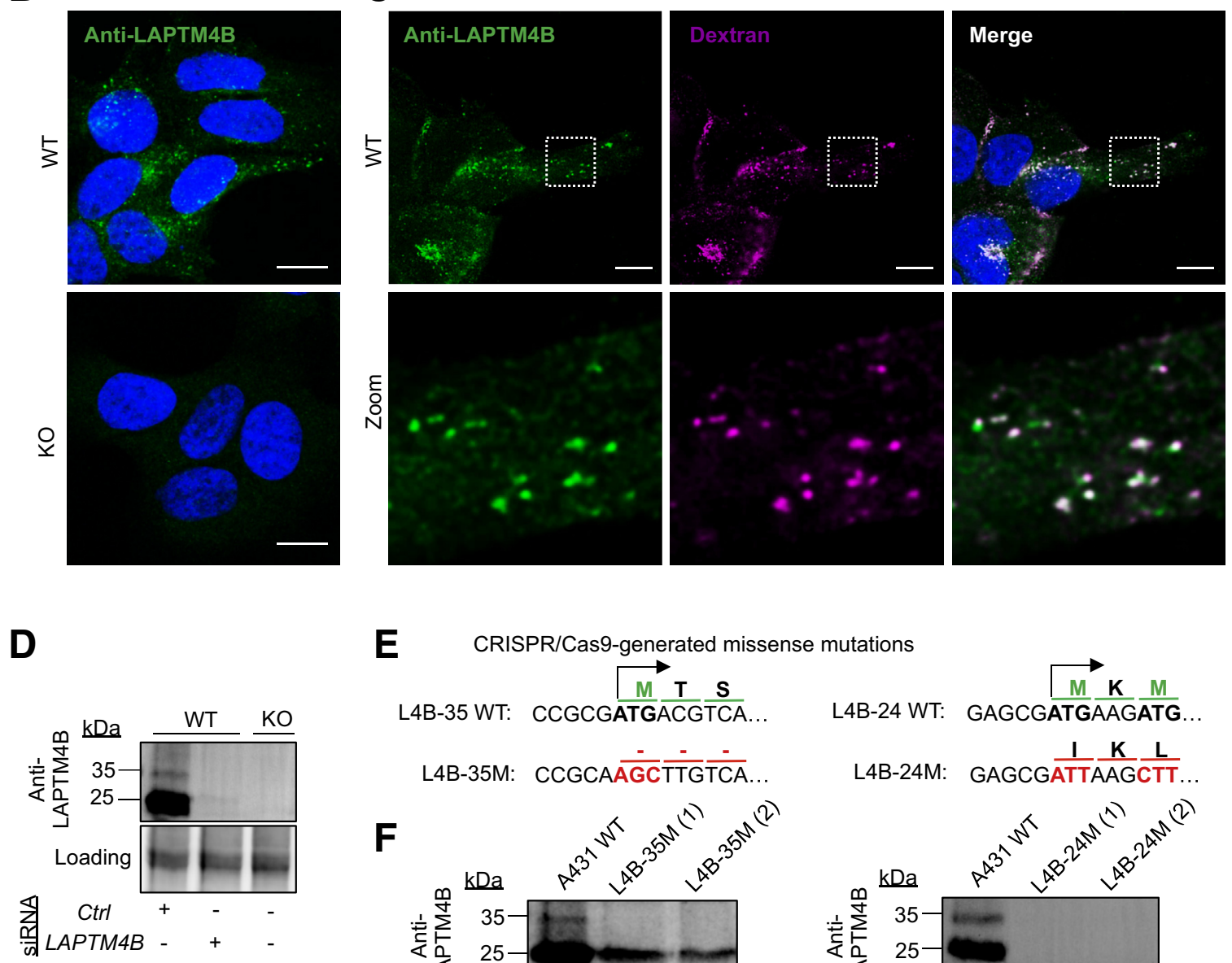

E CRISPR/Cas9-generated missense mutations

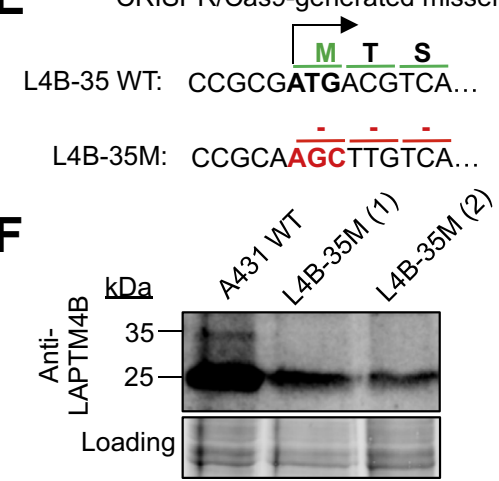

L4B-24 WT:

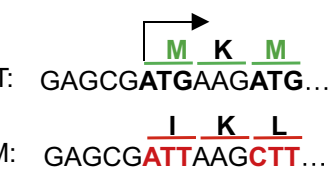

L4B-24M: GAGCGATTAAGCTT..

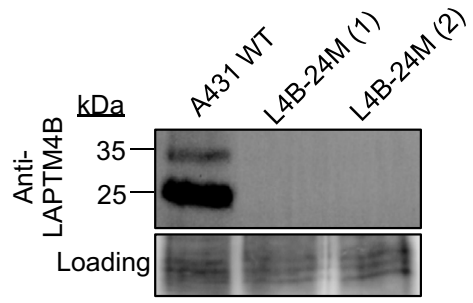

Figure 1 Characterization of the lysosome associated protein transmembrane 4B (LAPTM4B) antibody. A: Schematic overview of the LAPTM4B gene, transcript, and protein isoforms. The peptide used as an antigen is indicated between transmembrane domains 3 and 4. B: Wild-type (WT) A431 cells and LAPTM4B knockout (KO) cells are stained with LAPTM4B antibody. C: WT A431 cells were incubated with fluorescent dextran for 2 hours to label endosomal compartments (magenta), fixed, and stained with LAPTM4B antibody (green). Dotted box areas are shown in higher magnification in the bottom row. D: WT and LAPTM4B KO A431 cells were treated with the indicated siRNAs, and LAPTM4B expression was assessed by Western blotting. E: Schematic overview of CRISPR/Cas9-generated missense mutations of LAPTM $4 B-35$ and LAPTM4B-24 start codons. Green indicates the start codon sequence of $\angle A P T M 4 B$ isoforms, and red the mutated sequence. F: Western blot showing LAPTM4B isoform expression in cell lines with mutated LAPTM4B-35 start codon (L4B-35M, clones 1 and 2) or mutated LAPTM4B-24 start codon (L4B-24M, clones 1 and 2). Scale bars: $10 \mu \mathrm{m}$ (B and C).

inhibitor Cocktail (Sigma-Aldrich; catalog number P8340) in the ratio bead weight/sample weight/lysis buffer (1:1:2). The samples were homogenized twice using blender settings of speed 16 and time 4 . The lysates were collected and cleared by centrifugation 10 minutes at $11,800 \times g$, $4^{\circ} \mathrm{C}$. The samples were analyzed by Western blotting.
Human lung cancer tissue premade Western blot was from BioCat (Heidelberg, Germany; catalog number 1511-PS), human cancer tissues Western blots were from BioCat (catalog number 1542-PS, catalog number 1545-PS), and human healthy tissues Western blot membrane was from Novus Biologicals (Littleton, CO; catalog number NBP2-31378). 
A
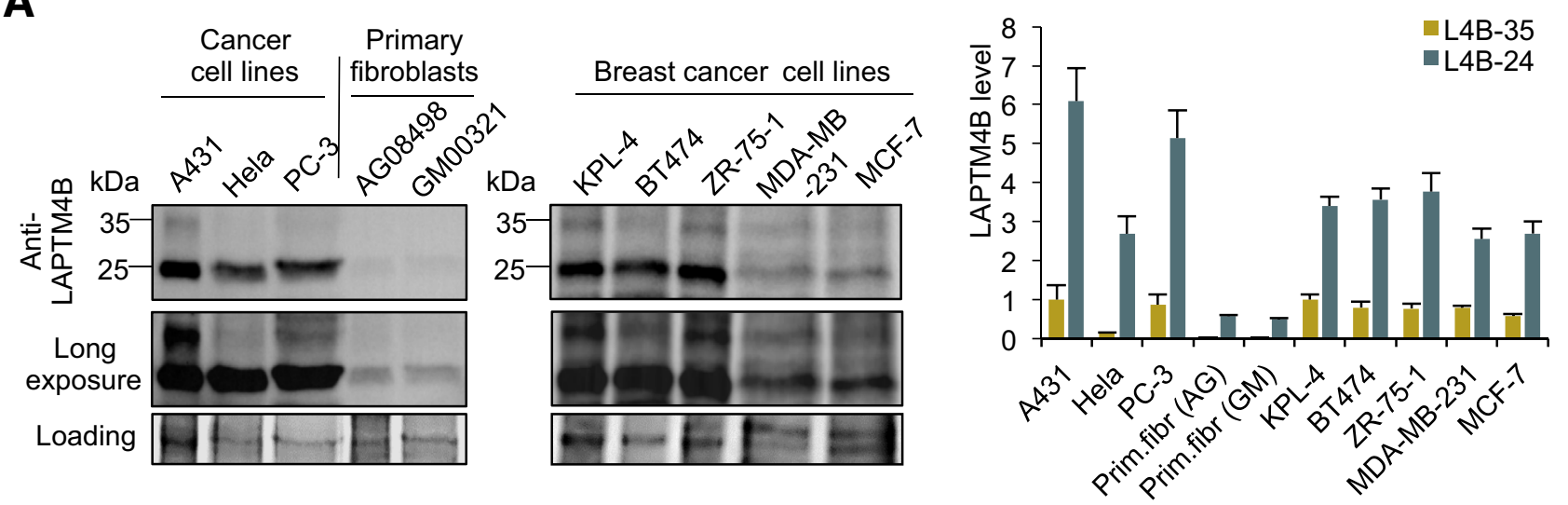

B
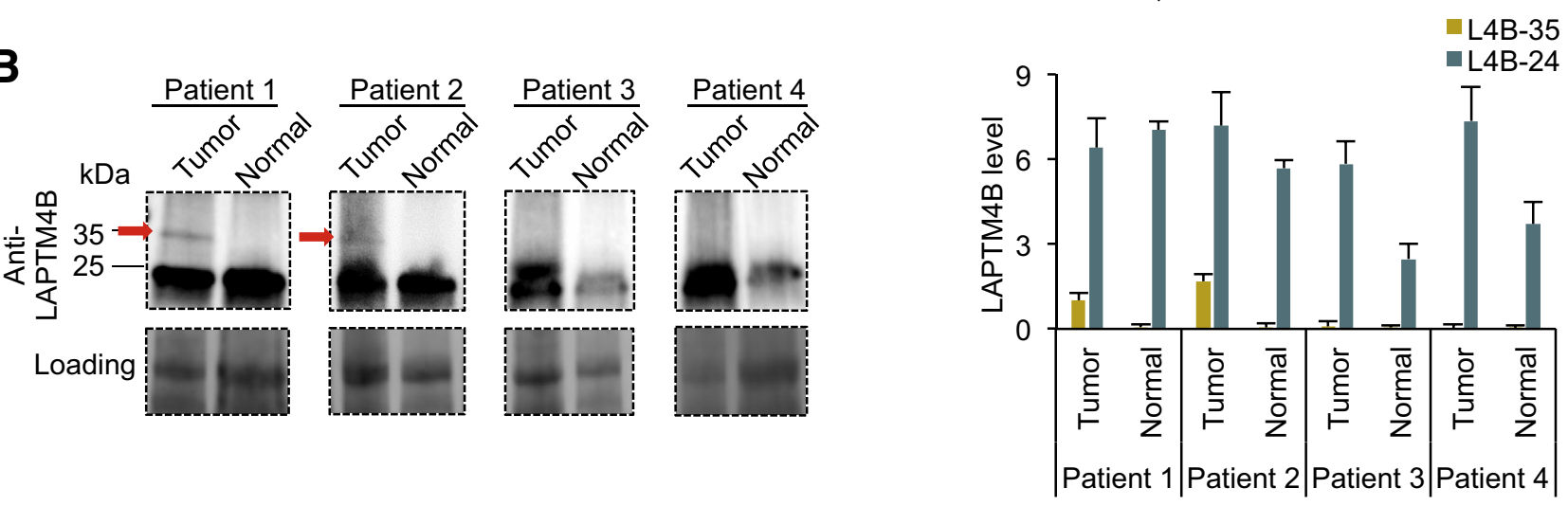

C
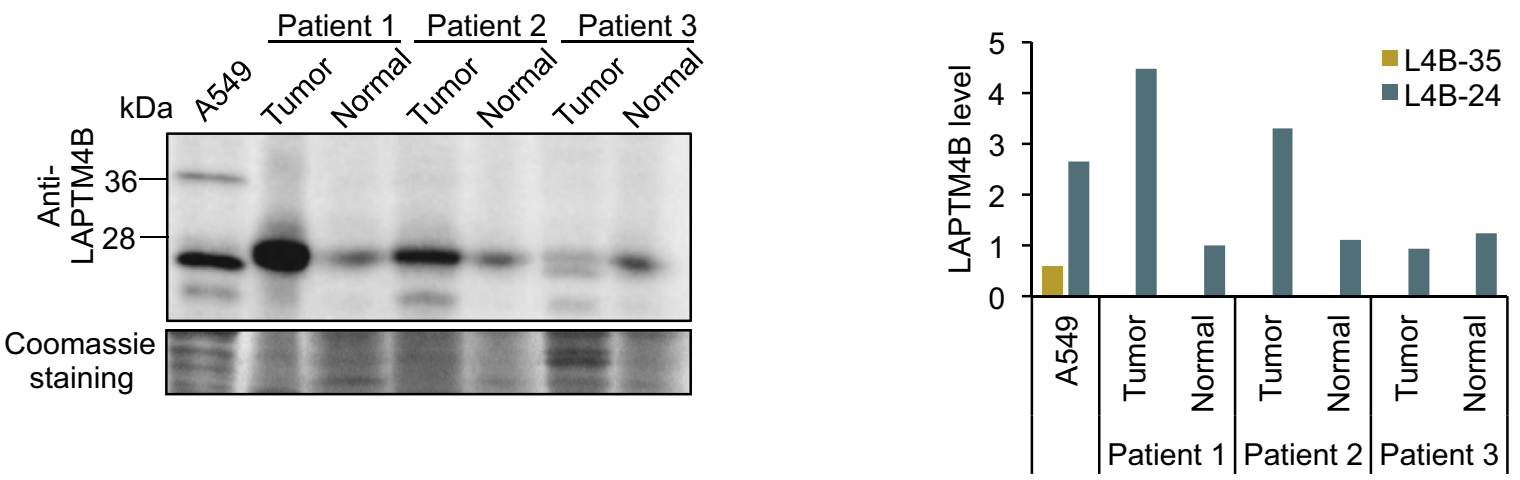

Figure 2 The lysosome associated protein transmembrane 4B (LAPTM4B)-24 is up-regulated upon malignant transformation. A: Cancer cell lines and healthy primary fibroblasts (Prim.fibr) were assessed for LAPTM4B expression by Western blotting. A total of $30 \mu \mathrm{g}$ of protein from different cell lines were loaded in each lane. Left panel: A representative experiment. Right panel: Quantification of $n=3$ experiments. B: LAPTM4B level in tumor tissue and paired adjacent non-tumor tissue from four breast cancer patients was assessed by Western blotting. A total of $30 \mu \mathrm{g}$ protein from different tissues was loaded per lane. Left panel: A representative experiment. Right panel: Quantification of $n=3$ technical repeats. Red arrows indicate the location of 35-kDa proteins on the Western blot. C: LAPTM4B level in tumor tissue and paired adjacent non-tumor tissue from three lung cancer patients, and in the lung cancer cell line A549, were assessed by Western blotting. A total of $15 \mu \mathrm{g}$ of protein from different tissues was loaded per lane. Right panel: Quantification of LAPTM4B levels with LAPTM4B-24 expression in Patient 1 normal tissue set as 1 . Data are expressed as means \pm SEM and are normalized to total protein content in the lane; and LAPTM4B-35 level in A431 cells (A), Patient 1 tumor sample (B), or Patient 1 normal tissue (C) was set as $1 . n=3$ experiments (A) and technical repeats (B).

\section{Statistical Analysis}

All of the data are presented as means \pm SEM from at least three independent experiments. Statistical significance was calculated using the $t$-test for pairwise comparisons. The level of statistical significance was set at 0.05 .

\section{Results}

\section{Characterization of the Anti-LAPTM4B Antibody}

To elucidate the expression of LAPTM4B isoforms in cells and tissues, a monoclonal LAPTM4B antibody was made under the antibody codevelopment program of Atlas 

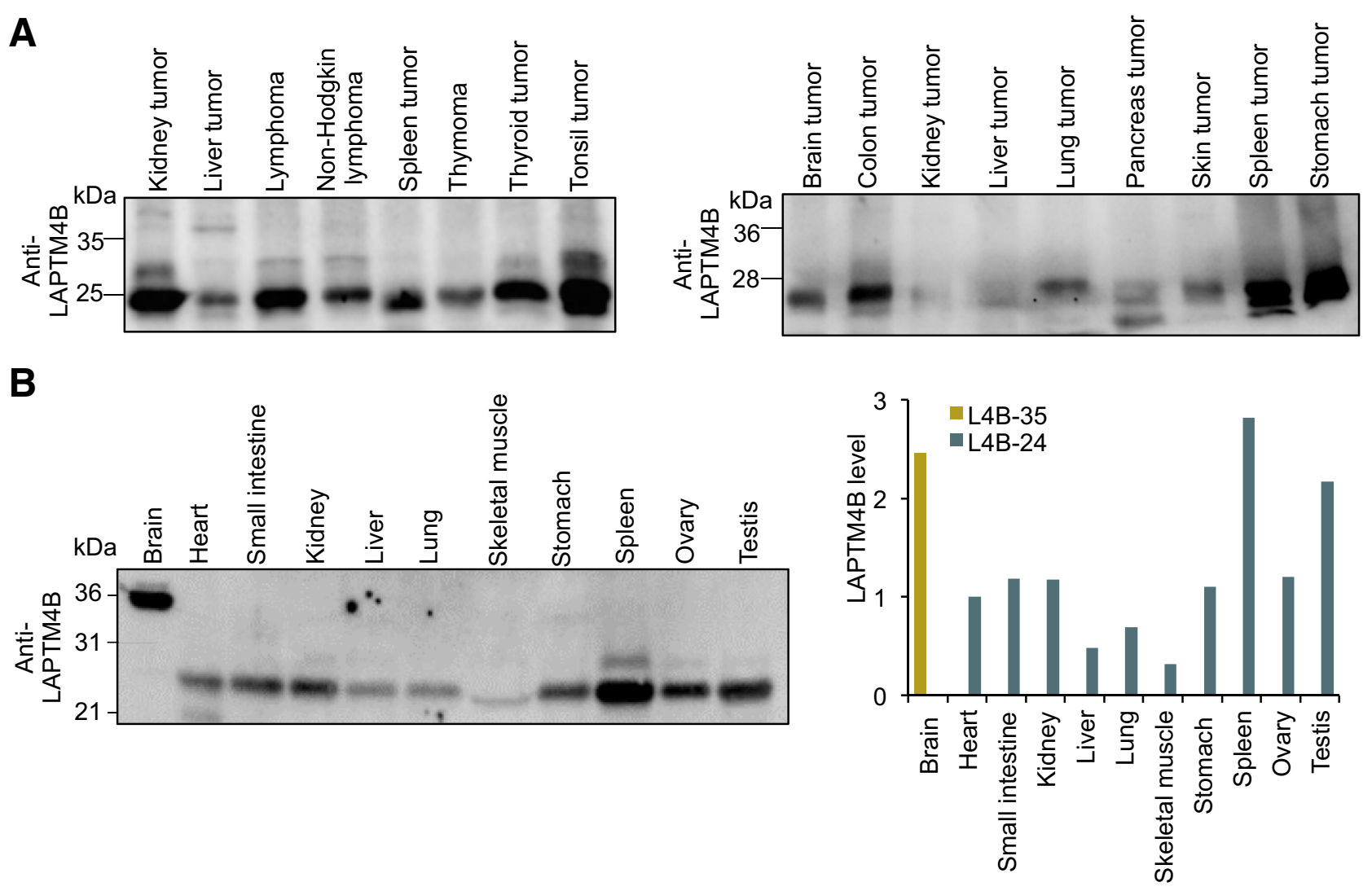

Figure 3 Lysosome associated protein transmembrane 4B (LAPTM4B) protein isoforms in normal and cancer tissues. A: LAPTM4B levels in tumor tissues from various origins are assessed by Western blotting. A total of $15 \mu \mathrm{g}$ of protein from different tumor tissues was loaded per lane. B: Left panel: LAPTM4B expression in healthy human tissues is assessed by Western blotting. A total of $50 \mu \mathrm{g}$ of protein from different tissues was loaded per lane. Right panel: Quantification of LAPTM4B expression with LAPTM4B-24 in heart set as 1.

Antibodies (Bromma, Sweden). The antigenic epitope is located in the loop connecting transmembrane domains 3 and 4 of human LAPTM4B and is therefore present in both isoforms (Figure 1A). In immunocytochemical stainings, the antibody detected punctate structures in wild-type but not in LAPTM4B KO A431 cells (Figure 1B). As expected, LAPTM4B immunoreactivity displayed a high degree of colocalization with dextran-labeled late endosomal compartments (Figure 1C). In Western blotting, the antibody detected a major 24-kDa and a minor 35-kDa band in A431 wild-type cells. These bands were absent in LAPTM4B KO cells and in cells treated with LAPTM4B siRNA (Figure 1D).

The authors next investigated whether the $24-\mathrm{kDa}$ and 35-kDa proteins stem from alternative translation start sites, or if they represent different post-translational modifications of the same protein. To this end, CRISPR/Cas9 and homology-directed repair were used to generate cell lines with targeted missense mutations that specifically interrupt either the predicted start codon for LAPTM4B-35 or LAPTM4B-24 (Figure 1E). In cells lacking the LAPTM4B$35 \mathrm{ORF}$, the $35-\mathrm{kDa}$ band was not expressed, but the 24-kDa protein was still detected, indicating that the two isoforms are translated from different start codons. In further support of this, the 24-kDa band was absent from cells with mutations in the predicted LAPTM4B-24 translation start site (Figure 1F). Of note, all of the mutant cell lines displayed reduced levels of LAPTM4B mRNA (Supplemental Figure S1). This agrees with the observation that transcripts with interrupted start codons are often subject to enhanced degradation. ${ }^{13}$ Together, these data indicate that the monoclonal antibody reliably detects LAPTM4B proteins by immunofluorescence microscopy and Western blotting, and verify the existence of two LAPTM4B isoforms translated from alternative start codons. Interestingly, A431 cells preferentially expressed LAPTM4B-24 rather than the expected $35-\mathrm{kDa}$ isoform that is encoded by the longest ORF or the transcript.

\section{LAPTM4B-24 Is the Predominant Isoform in Human Cell Lines and in Most Human Tissues}

Because LAPTM4B-35 has been associated with poor prognosis in cancers, ${ }^{8,14-18}$ the authors next assessed whether this isoform was specifically up-regulated in human cancer cell lines or primary tumor tissues. All of the tested cancer cell lines displayed strong expression of LAPT M4B-24 and low or undetectable levels of LAPTM4B-35 
A

$\begin{array}{lllllll}\text { Time (minutes) } & 0 & 20 & 40 & 60 & 120 & 180\end{array}$

Anti-LAPTM4B

Loading

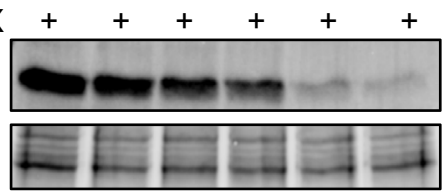

B

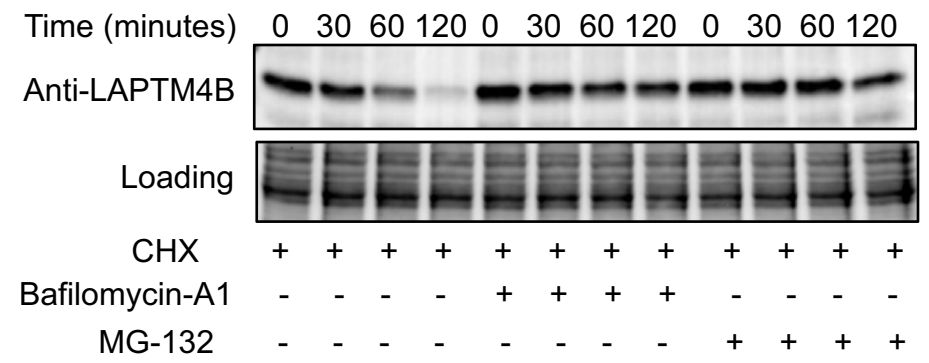

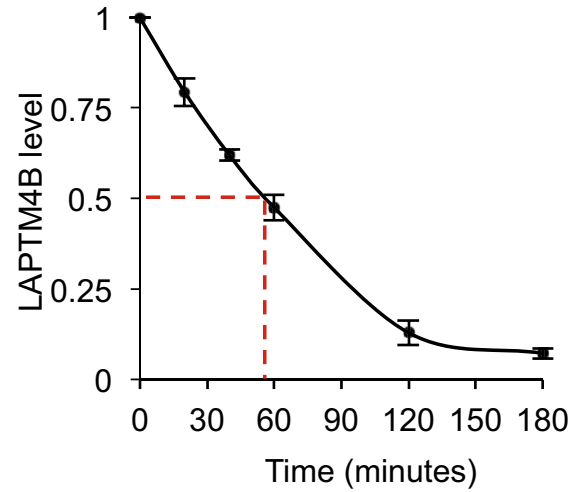

Time (minutes) $\quad 0 \quad 30 \quad 60120 \quad 0 \quad 30 \quad 60120$

Anti-LAPTM4B

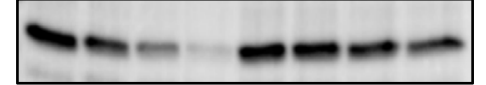

Loading

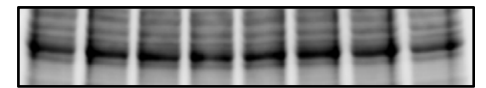

$\mathrm{CHX}$

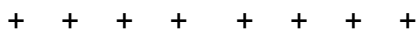

Bafilomycin-A1

MG-132
C

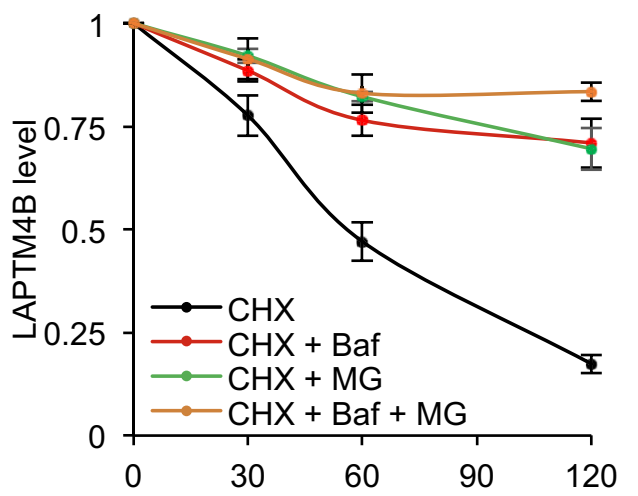

(Figure 2A). Intriguingly, in primary tumor tissue samples, LAPTM4B-35 expression was negligible or absent, and most tumors showed a predominant band at approximately $24 \mathrm{kDa}$ (Figure 2, B and C). LAPTM4B-24 was up-regulated in two of four breast cancer samples (Figure 2B) and two of three lung cancer samples compared with adjacent non-diseased tissues (Figure 2C). The predominant expression of the LAPTM4B-24 isoform was additionally observed in 17 tested tumor samples originating from 14 different tissue types (Figure 3A). Moreover, LAPTM4B-24 was the only isoform observed in healthy human primary fibroblasts and the majority of normal tissues examined (Figures $2 \mathrm{~A}$ and 3B). A notable exception is the brain, where only LAPTM4B-35 was detected (Figure 3B). However, datamining revealed that LAPTM4B-35 appears not to be essential in humans. The gnomAD database ${ }^{19}$ reports a frame shift mutation $(S 75 A f s)$ that specifically disrupts the ORF of LAPTM4B-35 while leaving LAPTM4B-24 intact
Figure 4 Lysosomal and proteasomal pathways are involved in the rapid turnover of lysosome associated protein transmembrane $4 \mathrm{~B}$ (LAPTM4B)-24. A: Wild-type (WT) A431 cells were treated with $50 \mu \mathrm{g} / \mathrm{mL}$ cycloheximide ( $\mathrm{CHX}$ ) for the indicated times, and endogenous LAPTM4B-24 expression is assessed by Western blotting. Left panel: A representative experiment. Right panel: Quantification of $n=3$ experiments. The red dashed line indicates the time point when half of the endogenous LAPTM4B-24 has been degraded. B: WT A431 cells were treated with 1 $\mu \mathrm{mol} / \mathrm{L}$ bafilomycin-A1 (Baf) and/or $20 \mu \mathrm{mol} / \mathrm{L}$ MG-132 (MG), together with $50 \mu \mathrm{g} / \mathrm{mL} \mathrm{CHX}$ for the indicated times, and endogenous LAPTM4B-24 expression is assessed by Western blotting. C: Quantification of data in (B). Data are expressed as means \pm SEM and are normalized to 0 minutes time point. $n=3$ experiments (A) and technical repeats (B); $n \geq 3$ independent experiments (C).

(Supplemental Figure S2A). The S75Afs allele is relatively frequent $(7.9 \%)$, and its homozygosity appears well tolerated and is in fact enriched among the oldest individuals in the database (Supplemental Figure S2B). The authors speculate that the selective expression of LAPTM4B-35 in the human brain might be related to maintaining sphingolipid homeostasis or specific nutrient requirements of the central nervous system. However, the lack of reported phenotypes in S75Afs homozygous individuals suggests that LAPTM4B-24 may compensate for LAPTM4B-35 function.

\section{LAPTM4B-24 Is Physiologically Important in Mice}

Of note, common laboratory animals including mouse, rat, and zebrafish do not encode an ORF for LAPTM4B-35 in their genomes, whereas LAPTM4B-24 is highly conserved between the species (Supplemental Figure S3). Moreover, Laptm 4b-24 KO mice show severe developmental defects 
A

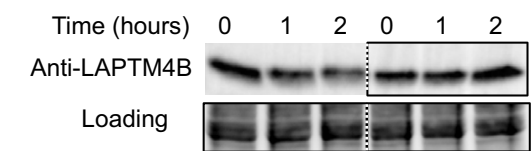

Bafilomycin-A1 - $-\quad-+++$

MG-132 - $\quad-\quad+\quad++$

EBSS ++++++

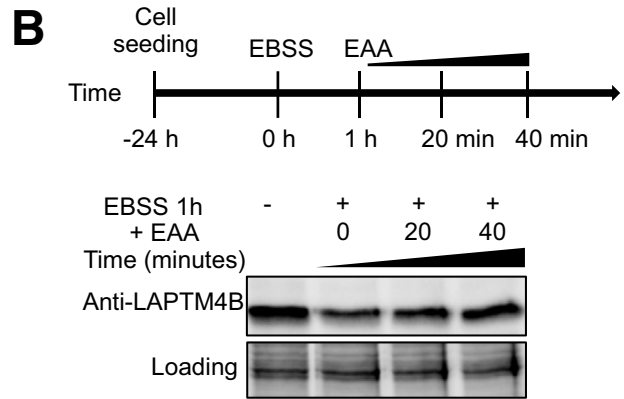

C

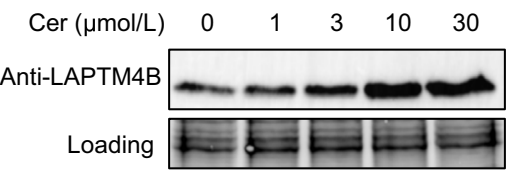

D

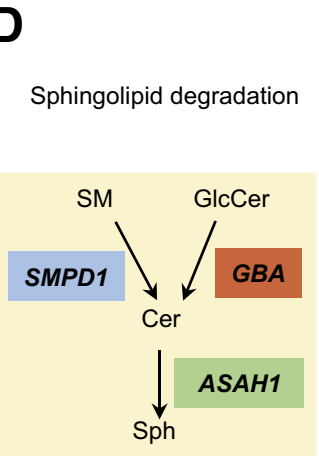

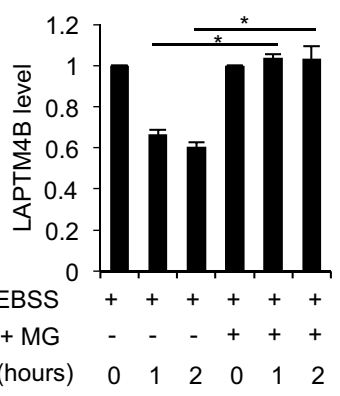
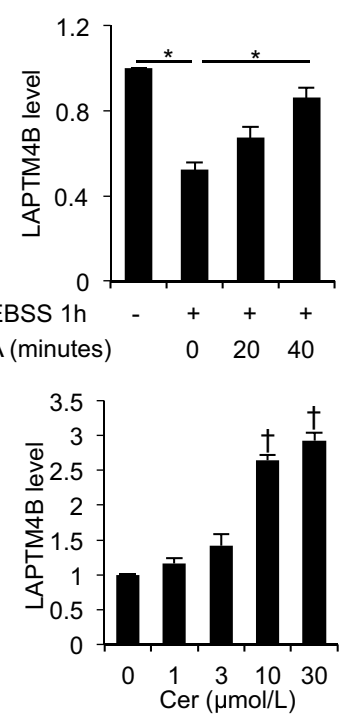

$\mathbf{F}$
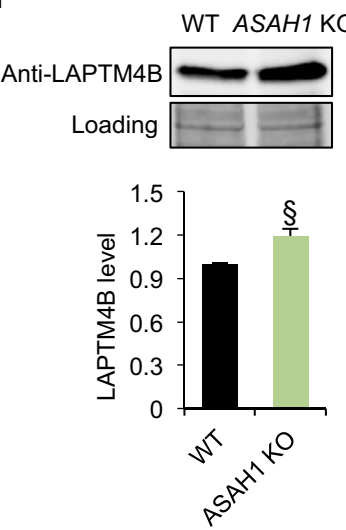

Figure 5 Lysosome associated protein transmembrane 4B (LAPTM4B)-24 protein levels are regulated by nutrient signaling and ceramide. $A$ : Wild-type (WT) A431 cells were treated with Earle Balanced Salt Solution (EBSS), with or without protein degradation inhibitors $\left[\begin{array}{ll}1 & \mu \mathrm{mol} / \mathrm{L}\end{array}\right.$ bafilomycin-A1 (Baf) and $20 \mu \mathrm{mol} / \mathrm{L}$ MG132(MG)], for the indicated times. Endogenous LAPTM4B-24 expression is assessed by Western blotting. Left panel: A representative experiment. Right panel: Quantification of $n=4$ experiments. B: WT A431 cells were starved 1 hour in EBSS and refed with stimulation medium for 20 minutes or 40 minutes. Endogenous LAPTM4B-24 expression is assessed by Western blotting. Left panel: Timeline of starving and refeeding cells, and a representative experiment. Right panel: Quantification of $n=3$ experiments. C: WT A431 cells were treated with indicated concentrations of C6ceramide (Cer) for 6 hours, and endogenous LAPTM4B-24 expression is assessed by Western blotting. Left panel: A representative experiment. Right panel: Quantification of $n=3$ experiments. D: The main lysosomal ceramide generating and degrading enzymes. E: WT A431 cells were treated with siRNAs against SMPD1 or GBA, and endogenous LAPTM4B-24 expression is assessed by Western blotting. Top panel: A representative experiment. Bottom panel: Quantification of $n=3$ experiments. F: Endogenous LAPTM4B-24 expression in WT and ASAH1 knockout (KO) cells was assessed by Western blotting. Top panel: A representative experiment. Bottom panel: Quantification of $n=4$ experiments. Data are expressed as means \pm SEM and are normalized to 0 hour time point $(\mathbf{A}$ and $\mathbf{B}), 0 \mu \mathrm{mol} / \mathrm{L}$ ceramide condition (C), Ctrl (E), or WT (F). $n=4$ experiments $(\mathbf{A}$ and $\mathbf{F}) ; n=3$ experiments ( $\mathbf{B}, \mathbf{C}$, and $\mathbf{E})$. ${ }^{\star} P<0.05 ;{ }^{\dagger} P<0.05$ versus $0 \mu \mathrm{mol} / \mathrm{L} ;{ }^{\ddagger} P<0.05$ versus control; ${ }^{\S} P<0.05$ versus WT. ASAH1, acid ceramidase; Cer, ceramide; EAA, stimulation medium; GBA, glucocerebrosidase; GlcCer, glucosylceramide; SM, sphingomyelin; SMPD1, acid sphingomyelinase; Sph, sphingosine. and die at the preweaning stage (International Mouse Phenotyping Consortium ${ }^{20}$ ), suggesting an essential function for LAPTM4B-24.

\section{LAPTM4B-24 and LAPTM4B-35 Display Different Subcellular Distributions and Functions}

Studies of LAPTM4B have frequently relied on overexpressing the protein. ${ }^{16,21-24}$ To dissect the functional properties of the individual isoforms, cells expressing either LAPTM4B-24 or -35 on a LAPTM4B knockout background were compared. The two isoforms displayed clear differences in their subcellular distribution. LAPTM4B-24 was predominantly endosomal, whereas LAPTM4B-35 was found both in endosomes and to a substantial degree in the plasma membrane (Supplemental Figure S4, A-D).

Because LAPTM4B has been shown to regulate cell migration $^{22,23}$ and mTORC1 signaling, ${ }^{6,25}$ the authors next assessed isoform-specific effects on these pathways. Labelfree real-time monitoring of cells using xCELLigence ${ }^{12}$ revealed that cell migration was stimulated by LAPTM4B35, but not by LAPTM4B-24 (Supplemental Figure S4E). 
Moreover, LAPTM4B-35 increased AKT phosphorylation at Ser 473, and this effect was not seen in LAPTM4B24-expressing cells (Supplemental Figure S4F). These results agree with previous studies showing that the PPRP motif in the N-terminus of LAPTM4B-35 regulates AKT and promotes cancer cell migration. ${ }^{4,22}$ However, the contribution of this pathway in tumor progression in vivo remains open, considering that LAPTM4B-35 was present only at low levels and in a small subset of tumors (Figures 2, $\mathrm{B}$ and $\mathrm{C}$, and 3A). To investigate LAPTM4B isoform effects on mTORC1 signaling, cells were starved for 1 hour with EBSS, and the phosphorylation of the mTORC1 substrates ribosomal protein S6 kinase B1 (S6K), eukaryotic translation initiation factor $4 \mathrm{E}$ binding protein (4EBP), and unc51 like autophagy activating kinase 1 (ULK1) were assessed upon refeeding with stimulation medium (complete medium/EBSS, 1:1). These experiments showed that S6K, 4EBP, and ULK1 phosphorylation was similarly stimulated by LAPTM4B-24 and -35 (Supplemental Figure S5). These data reinforce the notion that LAPTM4B functions in nutrition signaling. Accordingly, the main interaction partners of LAPTM4B are endosomal solute carriers and amino acid transporters, ${ }^{6,25}$ necessary for cellular metabolism.

\section{LAPTM4B-24 Is Degraded by Lysosomal and Proteasomal Pathways}

The new antibody was used in studying LAPTM4B-24 protein turnover. In A431 cells treated with the protein synthesis inhibitor cycloheximide, endogenous LAPTM4B24 was rapidly lost with a half-time of approximately 1 hour (Figure 4A). A similar result was obtained in cells stably overexpressing LAPTM4B-24 (Supplemental Figure S6A), suggesting that a post-translational mechanism is responsible for the rapid loss of LAPTM4B.

To dissect the pathways involved in endogenous LAPTM4B-24 degradation, cells were treated with the V-ATPase inhibitor bafilomycin-A1 to block lysosomal function, or with MG-132 to inhibit proteasomes. Both bafilomycin-A1 and MG-132 strongly attenuated LAPTM4B24 degradation in cycloheximide-treated cells (Figure 4, B and C). This indicates that both lysosomal and proteasomal pathways contribute to the turnover of endogenous LAPTM4B-24. Indeed, earlier data provided evidence that overexpressed LAPTM4B can be ubiquitinated by NEDD $4,{ }^{7}$ sorted into late endosomal compartments, ${ }^{9,26}$ and lysosomally degraded. ${ }^{27}$

\section{LAPTM4B-24 Is Regulated by Nutrient Availability and Ceramide}

Considering the rapid turnover of LAPTM4B and its role in mTORC1 signaling, ${ }^{6,25}$ it was next assessed whether endogenous LAPTM4B is regulated by nutrient availability. Indeed, starvation with EBSS induced a decrease in cellular LAPTM4B-24, which was blocked by inhibitors of lysosomal and proteasomal protein degradation (Figure 5A). The
LAPTM4B level was rapidly restored by refeeding stimulation medium to the starved cells (Figure 5B), indicating that LAPTM4B expression is responsive to nutrient availability. It has been previously shown that LAPTM4B interacts with ceramide, ${ }^{6,9}$ a simple sphingolipid regulating autophagy ${ }^{28,29}$ and mTORC1 signaling. ${ }^{6,30,31}$ The authors therefore hypothesized that ceramide may in part exert its effect by regulating LAPTM4B-24 expression. In support of this, C6-ceramide was found to increase endogenous LAPTM4B24 protein in a concentration-dependent manner (Figure 5C and Supplemental Figure S6, B and C). Instead, LAPTM4B24 protein level was not increased by the other lipids tested (cholesterol, oleic acid or C6-sphingomyelin) and was not markedly affected by glucose deprivation (Supplemental Figure S7), suggesting that ceramide exerts a specific effect in LAPTM4B regulation.

To investigate whether endogenously generated ceramide affects LAPTM4B-24 expression, A431 cells were depleted of lysosomal sphingolipid catabolizing enzymes (Figure 5D). Reducing lysosomal ceramide generation by knockdown of either acid sphingomyelinase (SMPD1) or glucocerebrosidase $(G B A)$ caused a decrease in cellular LAPTM4B (Figure 5E). Conversely, inhibition of lysosomal ceramide degradation by knockout of acid ceramidase (ASAHI KO) lead to moderately increased levels of LAPTM4B (Figure 5F). Together, this suggests that ceramide may exert a dual effect on LAPTM4B, by promoting its stabilization (this work) as well as by facilitating its interaction with lysosomal amino acid transporters to regulate mTORC1 signaling. ${ }^{6}$

\section{Discussion}

This study establishes the 24-kDa form of LAPTM4B that lacks the extreme $\mathrm{N}$-terminus of the protein as the predominantly expressed isoform in the majority of normal and malignant human cells and tissues studied. This study's results argue that the 35- and 24-kDa LAPTM4B isoforms are functionally distinct and demonstrate that in human cells, LAPTM4B-24 is subject to rapid turnover via both lysosomal and proteasomal degradation. LAPTM4B-24 is physiologically regulated by nutrient availability, with its levels being stabilized by lysosomal protein and lipid degradation products, that is, essential amino acids and ceramide.

Earlier studies have reported a strong association between LAPTM4B transcript levels and poor outcome in several cancers. ${ }^{3,32}$ Up-regulation of the LAPTM4B transcript has been observed in most carcinomas of the lung $(88 \%)$, colon $(67 \%)$, uterus $(83 \%)$, breast $(51 \%)$, and ovary $(69 \%){ }^{32}$ The detailed role of LAPTM4B in cancers remains unknown, and there are reports suggesting that both high ${ }^{3,33}$ and low $^{9,34}$ expression may promote chemotherapy resistance. An increasing number of studies point to a role for LAPTM4B in mTORC1 and autophagy signaling, and both mTORC1 activating $^{6,25}$ or autophagy promoting ${ }^{33,35}$ effects have been proposed. This suggests that LAPTM4B itself may not be a 
central regulator of autophagy and chemotherapy resistance, but rather exerts context dependent modulation of these processes. Interpretation of the available data is further complicated by the fact that studies frequently utilize overexpression of either the 24- or 35-kDa LAPTM4B isoform that exhibits in part distinct effects on cellular functions.

Although increased expression of LAPTM4B-35 has been proposed as a driving factor of cancer progression, our findings suggest that the expression of this isoform is low or negligible in most tumors. Thus, it seems plausible that upregulation of the $24-\mathrm{kDa}$ isoform may be responsible for many of the observed detrimental effects of LAPTM4B in cancers. Interestingly, recent studies have identified a minor polymorphic variant termed $L A P T M 4 B * 2$ that contains a 19-bp tandem repeat in the $5^{\prime}$ UTR. ${ }^{36,37}$ This allele has been shown to predict poor prognosis in malignant melanoma, colorectal and esophageal and prostate cancers. ${ }^{38-40}$ Whether this variant allele promotes the expression of LAPTM4B-35 will be interesting to address in future studies.

\section{Acknowledgments}

We thank HiLIFE Light Microscopy platform and Biocenter Finland imaging infrastructure (Biomedicum Imaging Unit) for technical support, the Genome Aggregation Database (GnomAD) and the groups that provided exome and genome variant data to this resource, and Juho Pirhonen for assistance with GnomAD data analysis.

\section{Author Contributions}

T.B., E.I., K.Z. conceptualized the project; K.Z., T.B., A.D. designed and performed experiments; K.Z., T.B. and E.I. wrote the manuscript with input from all authors; T.B. and E.I. acquired the funding and supervised the study.

\section{Supplemental Data}

Supplemental material for this article can be found at http://doi.org/10.1016/j.ajpath.2020.07.003.

\section{References}

1. Shao GZ, Zhou RL, Zhang QY, Zhang Y, Liu JJ, Rui JA, Wei X, Ye DX: Molecular cloning and characterization of LAPTM4B, a novel gene upregulated in hepatocellular carcinoma. Oncogene 2003, 22:5060-5069

2. Ng SWK, Mitchell A, Kennedy JA, Chen WC, McLeod J, Ibrahimova N, Arruda A, Popescu A, Gupta V, Schimmer AD, Schuh AC, Yee KW, Bullinger L, Herold T, Görlich D, Büchner T, Hiddemann W, Berdel WE, Wörmann B, Cheok M, Preudhomme C, Dombret H, Metzeler K, Buske C, Löwenberg B, Valk PJM, Zandstra PW, Minden MD, Dick JE, Wang JCY: A 17-gene stemness score for rapid determination of risk in acute leukaemia. Nature 2016, 540:433-437

3. Li Y, Zou L, Li Q, Haibe-Kains B, Tian R, Le Y, Desmedt C, Sotiriou C, Szallasi Z, Iglehart JD, Richardson AL, Wang ZC: Amplification of LAPTM4B and YWHAZ contributes to chemotherapy resistance and recurrence of breast cancer. Nat Med 2010, 16:214-218

4. Li L, Wei XH, Pan YP, Li HC, Yang H, He QH, Pang Y, Shan Y, Xiong FX, Shao GZ, Zhou RL: LAPTM4B: a novel cancer-associated gene motivates multidrug resistance through efflux and activating PI3K/AKT signaling. Oncogene 2010, 29:5785-5795

5. Tan X, Sun Y, Thapa N, Liao Y, Hedman AC, Anderson RA: LAPTM4B is a PtdIns(4,5)P2 effector that regulates EGFR signaling, lysosomal sorting, and degradation. EMBO J 2015, 34:475-490

6. Zhou K, Dichlberger A, Martinez-Seara H, Nyholm TKM, Li S, Kim YA, Vattulainen I, Ikonen E, Blom T: A ceramide-regulated element in the late endosomal protein LAPTM4B controls amino acid transporter interaction. ACS Cent Sci 2018, 4:548-558

7. Milkereit R, Rotin D: A role for the ubiquitin ligase Nedd4 in membrane sorting of LAPTM4 proteins. PLoS One 2011, 6:e27478

8. Meng Y, Wang L, Chen D, Chang Y, Zhang M, XU J-J, Zhou R, Zhang Q-Y: LAPTM4B: an oncogene in various solid tumors and its functions. Oncogene 2016, 35:6359-6365

9. Blom T, Li S, Dichlberger A, Bäck N, Kim YA, LoizidesMangold U, Riezman H, Bittman R, Ikonen E: LAPTM4B facilitates late endosomal ceramide export to control cell death pathways. Nat Chem Biol 2015, 11:799-806

10. Li S, Prasanna X, Salo VT, Vattulainen I, Ikonen E: An efficient auxin-inducible degron system with low basal degradation in human cells. Nat Methods 2019, 16:866-869

11. Pietiäinen V, Vassilev B, Blom T, Wang W, Nelson J, Bittman R, Bäck N, Zelcer N, Ikonen E: NDRG1 functions in LDL receptor trafficking by regulating endosomal recycling and degradation. J Cell Sci 2013, 126:3961-3971

12. Bird C, Kirstein S: Real-time, label-free monitoring of cellular invasion and migration with the xCELLigence system. Nat Methods 2009, 6. v-vi

13. Neymotin B, Ettorre V, Gresham D: Multiple transcript properties related to translation affect mRNA degradation rates in Saccharomyces cerevisiae. G3 (Bethesda) 2016, 6:3475-3483

14. Dong X, Tamura K, Kobayashi D, Ando N, Sumita K, Maehara T: LAPTM4B-35 is a novel prognostic factor for glioblastoma. J Neurooncol 2017, 132:295-303

15. Zhang H, Wei C, Liu R, Qi S, Liang P, Qi C, Wang A, Sheng B, Li L, Xu Y: Overexpression of LAPTM4B-35: a novel marker of poor prognosis of prostate cancer. PLoS One 2014, 9:e91069

16. Wang L, Meng Y, Xu J-J, Zhang Q-Y: The transcription factor AP4 promotes oncogenic phenotypes and cisplatin resistance by regulating LAPTM4B expression. Mol Cancer Res 2018, 16:857-868

17. Kang Y, Yin M, Jiang W, Zhang H, Xia B, Xue Y, Huang Y: Overexpression of LAPTM4B-35 is associated with poor prognosis in colorectal carcinoma. Am J Surg 2012, 204:677-683

18. Zhou L, He X-D, Cui Q-C, Zhou W-X, Qu Q, Zhou R-L, Rui J-A, Yu J-C: Expression of LAPTM4B-35: a novel marker of progression, invasiveness and poor prognosis of extrahepatic cholangiocarcinoma. Cancer Lett 2008, 264:209-217

19. Karczewski KJ, Francioli LC, Tiao G, Cummings BB, Alföldi J, Wang Q, et al: The mutational constraint spectrum quantified from variation in 141,456 humans. Nature 2020, 581:434-443

20. Dickinson ME, Flenniken AM, Ji X, Teboul L, Wong MD, White JK, et al: High-throughput discovery of novel developmental phenotypes. Nature 2016, 537:508-514

21. Cheng X, Zheng Z, Bu Z, Wu X, Zhang L, Xing X, Wang X, Hu Y, Du H, Li L, Li S, Zhou R, Wen XZ, Ji JF: LAPTM4B-35, a cancerrelated gene, is associated with poor prognosis in TNM stages I-III gastric cancer patients. PLoS One 2015, 10:e121559

22. Liu X, Xiong F, Wei X, Yang H, Zhou R: LAPTM4B-35, a novel tetratransmembrane protein and its PPRP motif play critical roles in proliferation and metastatic potential of hepatocellular carcinoma cells. Cancer Sci 2009, 100:2335-2340

23. Yang H, Xiong F, Wei X, Yang Y, McNutt MA, Zhou R: Overexpression of LAPTM4B-35 promotes growth and metastasis of hepatocellular carcinoma in vitro and in vivo. Cancer Lett 2010, 294:236-244 
24. Zhou L, He XD, Yu JC, Zhou RL, Yang H, Qu Q, Rui JA: Overexpression of LAPTM4B promotes growth of gallbladder carcinoma cells in vitro. Am J Surg 2010, 199:515-521

25. Milkereit R, Persaud A, Vanoaica L, Guetg A, Verrey F, Rotin D: LAPTM4b recruits the LAT1-4F2he Leu transporter to lysosomes and promotes mTORC1 activation. Nat Commun 2015, 6:7250

26. Tan X, Thapa N, Sun Y, Anderson RA: A kinase-independent role for EGF receptor in autophagy initiation. Cell 2015, 160:145-160

27. Vergarajauregui S, Martina JA, Puertollano R: LAPTMs regulate lysosomal function and interact with mucolipin 1: new clues for understanding mucolipidosis type IV. J Cell Sci 2011, 124:459-468

28. Jiang W, Ogretmen B: Autophagy paradox and ceramide. Biochim Biophys Acta 2014, 1841:783-792

29. Harvald EB, Olsen ASB, Færgeman NJ: Autophagy in the light of sphingolipid metabolism. Apoptosis 2015, 20:658-670

30. Hsieh C-T, Chuang J-H, Yang W-C, Yin Y, Lin Y: Ceramide inhibits insulin-stimulated Akt phosphorylation through activation of Rheb/mTORC1/S6K signaling in skeletal muscle. Cell Signal 2014, 26:1400-1408

31. Young MM, Kester M, Wang HG: Sphingolipids: regulators of crosstalk between apoptosis and autophagy. J Lipid Res 2013, 54: $5-19$

32. Kasper G, Vogel A, Klaman I, Gröne J, Petersen I, Weber B, Castaños-Vélez E, Staub E, Mennerich D: The human LAPTM4b transcript is upregulated in various types of solid tumours and seems to play a dual functional role during tumour progression. Cancer Lett 2005, 224:93-103

33. Li Y, Zhang Q, Tian R, Wang Q, Zhao JJ, Iglehart JD, Wang ZC, Richardson AL: Lysosomal transmembrane protein LAPTM4B promotes autophagy and tolerance to metabolic stress in cancer cells Cancer Res 2011, 71:7481-7489

34. de Ronde JJ, Lips EH, Mulder L, Vincent AD, Wesseling J, Nieuwland M, Kerkhoven R, Vrancken Peeters M-JTFD, Sonke GS, Rodenhuis S, Wessels LFA: SERPINA6, BEX1, AGTR1, SLC26A3, and LAPTM4B are markers of resistance to neoadjuvant chemotherapy in HER2-negative breast cancer. Breast Cancer Res Treat 2013, 137:213-223

35. Maki Y, Fujimoto J, Lang W, Xu L, Behrens C, Wistuba II, Kadara H: LAPTM4B is associated with poor prognosis in NSCLC and promotes the NRF2-mediated stress response pathway in lung cancer cells. Sci Rep 2015, 5:13846

36. Wang B, Xu J, Zhou R, Zhang Q: Association of LAPTM4B gene polymorphism with nasopharyngeal carcinoma susceptibility in a Chinese population. Med Oncol 2013, 30:470

37. Meng F, Song H, Luo C, Yin M, Xu Y, Liu H, Zhou R, Lou G: Correlation of LAPTM4B polymorphisms with cervical carcinoma. Cancer 2011, 117:2652-2658

38. Hashemi M, Rezaei M, Narouie B, Simforoosh N, Basiri A, Ziaee SAM, Bahari G, Taheri M: Association between LAPTM4B gene polymorphism and prostate cancer susceptibility in an Iranian population. Mol Cell Oncol 2016, 3:e1169342

39. Cheng X, Tian X, Wu X, Xing X, Du H, Zhou C, Zhang Q, Hao C, Wen X, Ji J: Relationship between LAPTM4B gene polymorphism and prognosis of patients following tumor resection for colorectal and esophageal cancers. PLoS One 2016, 11:e158715

40. Zhang M, Zhou R, Xu J, Zhang Q: Relationship between LAPTM4B gene polymorphism and susceptibility of malignant melanoma in Chinese patients. Transl Oncol 2014, 7:638-643 